\title{
Implementing guidelines for depression on antidepressant prescribing in general practice: a quasi-experimental evaluation
}

\author{
Gerdien Franx ${ }^{1 *}$, Jochanan Huyser ${ }^{2}$, Jan Koetsenruijter ${ }^{3}$, Christina M van der Feltz-Cornelis ${ }^{1,4,5}$, Peter FM Verhaak ${ }^{6,7}$, \\ Richard PTM Grol ${ }^{3}$ and Michel Wensing ${ }^{3}$
}

\begin{abstract}
Background: Internationally, guidelines for depression recommend a stepped care approach, implying that antidepressant medication should not be offered as a first step treatment to patients with sub-threshold or mild depression. In the Netherlands, antidepressant prescribing rates in general practice as a first treatment step are considered to be high. The aim of this study was to evaluate the implementation of guideline recommendations on antidepressant prescribing.

Methods: A quasi-experimental study with a non-equivalent naturalistic control group and three years follow-up was performed in the general practice setting in the Netherlands. General Practitioners (GPs) participated in a national Quality Improvement Collaborative (QIC), focusing on the implementation of a guideline based model for a stepped care approach to depression. The model consisted of self-help and psychological treatment options for patients with milder symptoms as an alternative to antidepressants in general practice. Changes in antidepressant prescription rates of GPs were documented for a three-year period and compared to those in a control group of GPs, selected from an ongoing national registration network.
\end{abstract}

Results: A decrease of $23.3 \%$ (49.4\%-26.1\%) in antidepressant prescription rates for newly diagnosed patients with depressive symptoms was found within the intervention group, whereas no difference occurred in the reference group (50.3\%-52.6\%). The decrease over time was significant, compared to the usual care group (OR 0.44, 95\% Cl: 0.21-0.92).

Conclusions: An implementation program using stepped care principles for the allocation of depression interventions resulted in reduced antidepressant prescription rates in general practice. GPs can change prescribing behaviour within the context of a QIC.

Keywords: General practice, Guidelines, Antidepressants, Implementation, Stepped care

\section{Background}

Depression is a highly prevalent condition with a range of effective treatment options, many of which can be offered in general practice. Since 2004, guidelines in several countries recommend a 'stepped care approach' as a framework for organising depression care, putting treatment options in a specific order and relating them to patient severity profiles [1-5].

\footnotetext{
* Correspondence: gfranx@trimbos.nl

${ }^{1}$ Trimbos Institute, Netherlands institute of mental health and addiction, PO Box 725, 3500 AS Utrecht, the Netherlands

Full list of author information is available at the end of the article
}

Derived from this framework, the national evidencebased multidisciplinary guideline for depression in the Netherlands, developed by a Guideline Development Group (GDG) consisting of GPs, psychiatrists, psychologists, allied health professionals, consumers and carers, recommended that antidepressant medication should not be offered as a first step treatment to patients with sub-threshold or mild depression. Instead, brief and non-pharmaceutical interventions including watchful waiting, (guided) self-help based on cognitive behavioural therapy (CBT), physical exercise and problem solving therapy were considered appropriate choices in the beginning of a treatment episode. Antidepressant medication or psychotherapy was to 
be offered as a first step treatment option to patients with moderate, severe or chronic symptoms [1,2].

Depression care, according to guideline recommendations, does not seem to be current practice. Rates of guideline-concordant care reported in the literature vary depending on setting, country and criteria for appropriateness, from $22 \%$ in the United States [6] to $42 \%$ in the Netherlands [7]. Focusing on recommendations concerning antidepressant prescribing, a number of problems exist. Firstly, antidepressant prescription rates in primary care are high in the Netherlands: $76 \%$ in 2002 and $70 \%$ in 2008 [8]. Although these rates appear to have declined in recent years, they have risen strongly over the last decades, with rises of more than $30 \%$ being reported in different countries [9-14]. One explanation for this rise of volume in antidepressant prescribing is the change in the proportion of patients receiving long term treatment $[15,16]$. Secondly, antidepressant prescription for depression during the first contact with the patient has also risen, from $62 \%$ of the cases in 1993 to $73 \%$ in 1998 [17]. Thirdly, there is a strong variation in prescribing between GPs, which can be explained by population, GP and practice characteristics [12]. Finally, prescription of antidepressants by GPs seems unrelated to symptom severity [18]. In addition, effective and brief, low intensity alternatives are relatively unknown as yet whereas it is considered essential that they are implemented in general practice [19]. As well, considering the fact that antidepressant treatment does not comply with the preferences of a great deal of patients, many of whom give negative reports of ineffectiveness and side-effects, there seems to be a need to change prescribing behaviour in general practice in the direction of a more stepped care approach, and in accordance with the clinical guidelines [18,20-23].

In order to bring about this change and implement key recommendations of the national depression guidelines in the Netherlands, a QIC with a three year follow-up was run from December 2006 until March 2008, as part of the National Depression Initiative [24]. QICs are multifaceted implementation strategies, offered to clinical care teams to rapidly improve performance and outcomes [25-28]. Parallel to the QIC, an implementation study was performed to determine the impact of the Depression QIC on antidepressant prescribing by GPs.

\section{Methods}

Adopting a stepped care model for the management of depression is a major change and thus makes it difficult to allocate randomly to healthcare professionals, because of the risk of low inclusion rates and withdrawal at professional level because of discontentment with the randomization procedure. Therefore we performed a quasi-experimental evaluation with a non-equivalent naturalistic control group and a three year follow-up period.

\section{Study population}

The study included two study groups ('QIC' and 'usual care', see below) and three measurement moments in each group. The health professionals in the intervention group were GPs (who provide all primary medical care in the Netherlands) participating in the depression QIC programme, described in detail elsewhere [29]. At the start of the QIC, all thirty-nine GPs were invited to participate in the study, alongside their implementation work. In order to participate, they had to consent to comply with data collection procedures. Practices were paid a fee for the time spent on research activities. Finally, twenty GPs consented and were included in the study.

The control group included GPs from practices participating in the Netherlands Information Network of General Practice (LINH), the principle national database in the Netherlands for general practice research, holding longitudinal and nationally representative data on morbidity, prescribing, and referrals [30]. In principle, patients receiving care as usual, had access to all types of depression treatment, including the low intensity treatments, although these were relatively unknown by primary care providers [19]. LINH physicians and the QIC physicians were considered to be proper naturalistic comparison groups, since participation in both programmes required the GPs' commitment to register practice data for research and quality improvement purposes. LINH-practices were only included in the study if the Electronic Medical Record (EMR) provided information about at least $90 \%$ of the three years study duration.

The included patients in both groups were aged 1865 , with a newly recorded diagnosis of depression as documented by the GPs in the EMR, along with an International Classification of General Practice (ICPC) diagnosis of depressive feelings (ICPC code P03) or depression (ICPC code P76) [31-33].

Ethical approval was provided by the METIGG, a national ethics committee in mental healthcare in the Netherlands.

\section{Intervention}

A Depression QIC was executed over fifteen months. A QIC is an implementation strategy applied in many countries for various clinical problems, and generally has five essential features: (1) a focus on a specific topic with gaps between best and current practice; (2) clinical experts providing ideas and support for improvement; (3) multidisciplinary teams from multiple sites participate; (4) there is a model for improvement (setting targets, collecting data and testing changes); and (5) a collaborative process with a series of structured activities in a given time frame [28,34,35]. These structured activities, which were offered to the participants during the Depression QIC, are listed in Table 1.

The focus of the Depression QIC was a stepped care model for depression treatment (see Figure 1), developed 
Table 1 Structured activities of the depression QIC Structured activities offered

- A network of multidisciplinary teams

- An expert team, teaching the stepped care model;

- SMART goal setting, a set of indicators to monitor results and an excel worksheet:

- A training for local team coordinators on breakthrough method and data collection;

- Four conference days for all improvement teams for exchange and learning;

- One conference day for local team coordinators for intensive exchange with the expert team;

- $\quad$ Five meetings between local team coordinators, with the expert team present;

- Team visits of experts and national project coordinators;

- Telephone contact between local and national coordinators;

- Written feedback on improvement reports and data charts;

- A virtual network environment for exchange of best-practices, a Toolkit of instruments and treatment protocols, online discussions and links to relevant sites;

- A two days training problem solving treatment for professionals;

- A workshop workflow Improvement.

by the QIC's clinical expert team and based on both the multidisciplinary guidelines and previous projects $[1,36,37]$. The model consisted of two pathways for patients with different severity profiles. Severity criteria were derived from the Diagnostic and Statistical Manual of Mental Disorders Fourth Edition (DSM IV) and based on the expert team's opinion. Antidepressant medication was not an option in treatment pathway 1 , but could be considered after a first step intervention had not resulted in sufficient treatment response. Antidepressants and psychotherapy were first line treatment options in pathway 2 . The model served to guide clinicians in their improvement work.

\section{Data collection}

The primary outcome was antidepressant prescribing, defined as the volume of antidepressant prescriptions for the depressed general practice population (prescription rates), issued by GPs as a first line treatment choice within one month after the diagnosis. The secondary outcome was referral by the GPs to clinicians providing psychological treatment. In both groups, all relevant data of patients with ICPC P03 or ICPC P76 were extracted from the EMRs of the general practices. Documentations by the physicians of co-morbid anxiety, using ICPC codes P01 (anxious, nervous, tensed feelings), P74 (anxiety disorder, condition of anxiety) and P75 (hysteria, hypochondria), were also extracted. Data extraction in the QIC group was performed by the physicians' assistants who had received a detailed protocol for computerised searching and support from the researchers. Data extraction in the control group was performed by the staff from the LINH database.

Antidepressant medication covered the subgroup N06A of the Anatomical Therapeutic Chemical (ATC) Classification System of the World Health Organization. Referrals included a documentation of a referral to a primary care psychologist or a specialised psychologist, a psychiatrist, a psychotherapist, an institution for ambulatory care or a mental health hospital. Data collection covered a three year period: the year 2006 indicates the baseline measurement before the QIC, the year 2007 indicates the year of the intervention and the year 2008 largely indicates the follow-up measurement, after the QIC had ended.

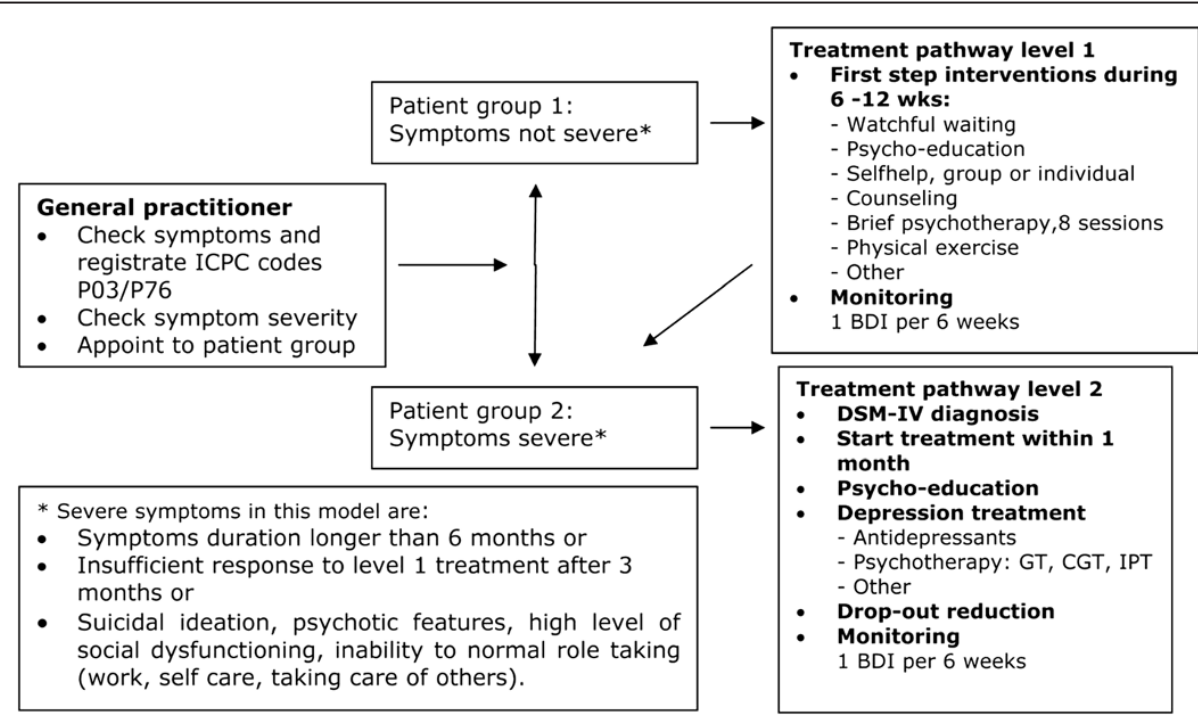

Figure 1 Stepped care depression model. 


\section{Data analysis}

Descriptive statistics were calculated within the groups. Using a t-test, we examined the changes during the three year follow-up within both study groups. To determine the effect of participation in the implementation programme and to correct for the clustering effect, we applied a multilevel logistical regression analysis with a two-level structure with patients nested within general practices. A statistical analysis was performed in MLwiN 2.15 comparing the outcomes between the two conditions, with antidepressant prescription (yes or no) or referral to mental healthcare (yes or no) as outcomes, and the following predictors to test the difference in changes between the two conditions: group (QIC or usual care), patient age and gender, co-morbid anxiety (yes or no ICPC P01, P74, P75), year $(2006,2007,2008)$ and an interaction term with year and group.

\section{Results}

Twenty GPs from seventeen practices participated in the intervention group, and 115 GPs from forty-one practices were selected as controls. In the intervention group, the data of 400 patients were extracted for analysis, and in the control group this number was 3956.

\section{Characteristics of the patient population}

In the QIC group, the mean age of patients with an ICPC documentation of P03 and P76 was 39.8 years, and 41.9 years in the control group. In the QIC group, the proportion of younger persons was higher (37.5\% versus $32.3 \%)$, whereas the control group consisted of a larger proportion of older persons (23.3\% versus 29.2\%) (Table 2).

\section{Antidepressant prescription}

Table 3 shows the changes in professional performance within both groups in terms of antidepressant prescribing and referrals. During the three year follow up, a decrease of $23.3 \%$ in the prescription of antidepressant medication occurred in the QIC group (from 49.4\% in 2006 to $26.1 \%$ in 2008). The usual care group did not change prescription rates (from $50.3 \%$ in 2006 to $52.6 \%$ in 2008 ).

Table 2 Characteristics of the patient population (in percentages)

\begin{tabular}{lccc}
\hline & $\begin{array}{c}\text { QIC practices } \\
(\mathbf{n}=\mathbf{4 0 0})\end{array}$ & $\begin{array}{c}\text { Usual care practices } \\
(\mathbf{n}=\mathbf{3 9 5 6})\end{array}$ \\
\hline Age & $18-35$ & 37.5 & 32.3 \\
& $36-50$ & 39.3 & 38.5 \\
& $51-65$ & 23.3 & 29.2 \\
& Mean & 39.8 & 41.9 \\
Male & 30.0 & 35.1 \\
Female & 70.0 & 64.9 \\
Comorbid anxiety & 4.0 & 5.8 \\
\hline
\end{tabular}

Table 3 Patients with first or new depressive symptoms receiving an antidepressant prescription or being referred to mental healthcare within one month (in percentages of the total of patients with a first or new depressive episode)

\begin{tabular}{lccccccc}
\hline & \multicolumn{3}{c}{ QIC practices } & & \multicolumn{3}{c}{ Usual care practices } \\
\cline { 2 - 4 } & $\mathbf{2 0 0 6}$ & $\mathbf{2 0 0 7}$ & $\mathbf{2 0 0 8}$ & & $\mathbf{2 0 0 6}$ & $\mathbf{2 0 0 7}$ & $\mathbf{2 0 0 8}$ \\
\hline $\begin{array}{l}\text { Antidepressant } \\
\text { prescription }\end{array}$ & 49.4 & $32.2^{*}$ & $26.1^{*}$ & & 50.3 & 47.0 & 52.6 \\
$\begin{array}{l}\text { Referral to mental } \\
\text { healthcare }\end{array}$ & 11.5 & 16.4 & 11.2 & & 10.1 & $13.0^{*}$ & 9.0 \\
$\mathrm{~N}$ & 87 & 152 & 161 & & 1261 & 996 & 1699 \\
\hline
\end{tabular}

*sign. < 0,05 compared with baseline (2006).

\section{Referral rates}

Overall, referral rates of GPs in the QIC practices were somewhat higher than in the usual care practices during the three years study interval. In 2006, $11.5 \%$ of the patients in QIC practices were referred within a month after diagnosis to a psychologist, a primary care psychologist, a psychiatrist, a psychotherapist, an institution for ambulatory care or a mental health hospital. In 2008, this rate remained at the same level of $11.2 \%$. With regards to baseline referral by GPs, in the usual care practices, a non-significant decline occurred from $10.1 \%$ in 2006 to $9 \%$ in 2008 (Table 3).

\section{Factors associated with antidepressant medication and} referral to mental healthcare

Table 4 shows the factors associated with the changes between the two study groups during our study period. The usual care clinicians did not change their prescribing behaviour in 2007 (OR 0.92) or 2008 (OR 0.87). In the QIC group, antidepressant prescribing as a first line treatment option did not change in 2007, but the frequency of prescribing decreased in 2008 in almost half of the cases,

Table 4 Factors associated with antidepressant prescribing and referral to mental healthcare

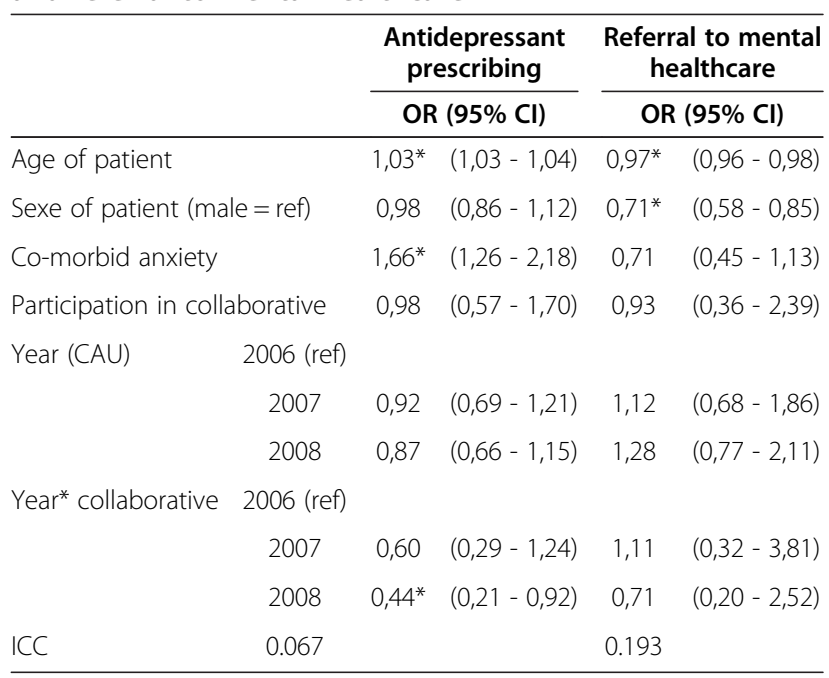

${ }^{*} \mathrm{p}<0,05, \mathrm{n}=4356$. 
compared to the usual care group (OR 0.44). QIC GPs tended to prescribe more frequently to older patients and to those who had additional anxiety problems. Multivariate analysis, however, showed that these characteristics did not account for the effect of the intervention and that participation in the QIC over time accounted for a significant decline in prescription rates (OR 0.44), compared to the usual care group. There was no significant change of referral behaviour in either group.

\section{Discussion and conclusions}

\section{Summary}

We found a substantial change in the professional performance of GPs participating in a quality improvement programme, in terms of lowered antidepressant prescription rates as a first step treatment choice for patients with depression. GPs providing usual care did not change their prescribing behaviour during the three year course of the study. In both groups, there was no change in referral rates to mental health clinicians.

The results seem to support the hypothesis that a QIC aimed at adherence to depression guidelines reduces antidepressant prescription rates of GPs, whereas GPs who have access to guidelines but who do not receive an intervention aimed at their implementation, don't change their prescribing behaviour. Two other qualitative studies, performed parallel to this controlled study, showed that instead of medication, the GPs started to offer low-intensity interventions to their patients during the QIC, such as guided self-help or brief psychotherapy, and that because of these alternative treatment options they felt more at ease in reducing antidepressant prescriptions for patients with mild symptoms $[29,38]$. The second result of the study, the lack of a significant change in referral rates in both groups, could point at the fact that the QIC participants did not replace the medication by a more specialised psychological intervention by a primary care psychologist or a specialist in the mental health sector, but by an intervention in general practice or by 'watchful waiting', namely psycho-education and pro-active follow up.

\section{Strengths and limitations}

A particular strength of this study was the evaluation of an ambitious quality improvement initiative with a direct comparison between two naturalistic groups, which makes the study appropriate to be included in an evidence review of quality improvement interventions [39]. Other strengths included the large numbers of patients and the substantial effect on the primary outcome.

A first study limitation was the lack of a randomisation procedure, which was not an option since the researchers had no control over the allocation of GPs to a particular condition. By conducting a randomised clinical trial, effects in terms of causality would have been stronger supported. However, RCTs may have the disadvantage of low inclusion rates and withdrawal at professional level because of discontentment with the randomization procedure, especially in implementation studies, thus introducing other problems of selection bias and lack of generalisibility of the results. Therefore, we considered the quasiexperimental design of this study valid for the exploration of our research question and dealt with this risk of selection bias by choosing the best possible comparison group in the Netherlands. This national database of GP performance, considered as the 'golden standard' for measuring care as usual because of the adequate documentation by these doctors who sign an agreement to document ICPC diagnosis and treatments provided. Unfortunately, this did not enable us to use patient reported depression outcomes, since these were not documented in the databases of routinely collected clinical data.

Another well known challenge in observational studies is the risk of bias due to confounding, which in our study could have occurred in terms of factors other than the QIC causing the observed changes. We were able to control for age, gender and co-morbid conditions of both study groups, but other factors may have played a role as well. Nevertheless, it is improbable that any one of these other factors would have caused a decline of $23 \%$ in prescription in the intervention group, which is considered to be quite substantial in the implementation literature. A second limitation of our study was the EMR data-extraction which was performed by administrative assistants in the different practices, who were very well known with the systems. We limited this risk of bias by providing all persons with the same instructions and by performing all data analysis by one research group.

\section{Comparison with existing literature}

Our study can be compared to the Depression QIC, organised by the Institute for Healthcare Improvement in the United States in 2000-2001 and based on Wagner's Chronic Care Model (CCM) [40]. The American QIC, also involving seventeen general practices, led to successful changes in the depression delivery and information system, which were also the most often sustained over time [41]. Organisational structure and leadership support were the most common facilitators, while staff resistance, time constraints, and information technology were the most common barriers.

Our study also relates to several initiatives in the United Kingdom to implement the guidelines of the National Institute for Health and Care Excellence (NICE). The Scottish study, 'Doing Well', incorporated the routine use of a depression severity measure with continuous outcome monitoring, a prompt access to guided self-help and a 'step-up' to more formal psychological therapy or medical care, if indicated. As a result, daily doses of 
antidepressants increased less rapidly than in other areas [42]. A British implementation study into stepped care services reported a considerable variation in the design and implementation of the stepped care guideline recommendations [43]. The large scale guideline implementation, Improving Access to Psychological Therapies (IAPT), focused on increasing the availability of evidence-based psychological treatments, both the high intensity therapies (CBT) and the low intensity therapies such as guided selfhelp, psycho-education groups and behavioural activation [44]. Three year results showed that most patients received guideline-concordant care and that patients had a higher chance of recovery if the treatment sites showed higher step-up rates from low to high intensity treatment in case of insufficient response, as well as if they received an adequate number of sessions [44].

\section{Implications for research and practice}

This study has shown that antidepressant prescribing by GPs can be changed by a multifaceted implementation strategy based on national guidelines and the time and support to implement these in a multidisciplinary context. This message is relevant for clinicians, managers and policy makers, both in Europe and beyond, who are motivated to implement guidelines for depression and to move from an overemphasis on psychopharmacological treatments for depression [45] to stepped depression care, where patients with mild symptoms receive less intensive treatments, such as medication. Policy initiatives aimed at strengthening general practice and reducing unnecessary antidepressant treatment in general practice can use our information, by addressing GPs, psychologists, social workers and specialised mental health nurses to recognise, treat and monitor depression in a stepped care manner, offering guided self-help and brief interventions when possible, and antidepressant medication when necessary [46]. Researchers charged with the task of evaluating such programs are recommended to consider adopting a randomised controlled design, to enable stronger statements about the effect of stepped care approaches and a useful cost effectiveness analysis. Although recruitment of participants to this type of implementation study is challenging, if feasible at all, useful frameworks exist to guide researchers in developing and evaluating these complex interventions [47].

\section{Conclusions}

GPs can learn to change antidepressant prescribing behaviour in the context of an improvement programme. Our study should be considered as one of the first studies focusing on the issue of the over prescribing of antidepressant treatment in general practice. It presents data indicating that GPs can change prescribing behaviour, provided that they have access to alternatives and implementation support. Future implementation studies should expand on this and investigate the stepped care delivery of all depression treatments, recommended in the guidelines. Fortunately, in the Netherlands and beyond, implementation of clinical guidelines followed by process and outcome monitoring for depression are gradually becoming mandatory and better supported by information technology. This is a hopeful message for those trying to improve the care for this patient group.

\section{Abbreviations}

ATC: Anatomical therapeutic chemical; CBT: Cognitive behavioural therapy; CCM: Chronic care model; DSM IV: Diagnostic and statistical manual of mental disorders fourth edition; EMR: Electronic medical record; GDG: Guideline development group; GP: General practitioner; ICPC: International classification of general practice; LINH: Netherlands information network of general practice; METIGG: National ethics committee in mental healthcare; NICE: National institute for health and care excellence; QIC: Quality improvement collaborative.

\section{Competing interest}

The authors declare that they have no competing interests.

\section{Authors' contributions}

GF carried out the design of the study, the acquisition, analysis and interpretation of data and the draft of the manuscript. JK carried out the statistical analysis of the data and participated in the draft of the manuscript. $\mathrm{JH}, \mathrm{CvdF}$ and PV have been involved in data interpretation and revising the manuscript for important intellectual content. RG and MW have made substantial contributions to the design of the study, the analysis and interpretation of data and the revision of the manuscript. All authors read and approved the final manuscript.

\section{Acknowledgements}

The data used in this study were made available by the primary care physicians participating in the study. We thank all of them, as well as the other clinicians participating in the study, their assistants and staff. This study was part of the Depression Initiative, a nationwide initiative lead by the Trimbos Institute to improve depression care according to the guidelines for depression. The Depression Initiative started in 2006 and ended in 2012. The study was funded by the Healthcare Insurance Innovation Fund (Innovatiefonds Zorgverzekeraars) and the Netherlands organisation for Health Research and Development (ZonMw, grant number 10-000-1002). Open access publication was financially supported by the Netherlands Organisation for Scientific Research (NWO).

\section{Author details}

${ }^{1}$ Trimbos Institute, Netherlands institute of mental health and addiction, PO Box 725, 3500 AS Utrecht, the Netherlands. ${ }^{2}$ Arkin, PO Box 75848, 1070 AV Amsterdam, the Netherlands. ${ }^{3}$ Scientific Institute for Quality of Healthcare, Radboud University Nijmegen Medical Centre, PO Box 9101, 114, 6500 HB Nijmegen, the Netherlands. ${ }^{\top}$ Tilburg University, Tranzo, Tilburg School of Social and Behavioral Sciences, PO Box 90153, 5000 LE Tilburg, the Netherlands. ${ }^{5}$ Clinical Centre for Body, Mind and Health, GGz Breburg, PO Box 770, 5000 AT Tilburg, the Netherlands. ${ }^{6}$ Nivel, Netherlands institute for health services research, PO Box 1568, 3500 BN Utrecht, the Netherlands. ${ }^{7}$ Rijksuniversiteit Universiteit Groningen, University Medical Centre Groningen, dep. General Practice, PO Box 196, FA20, 9700 AD Groningen, the Netherlands.

Received: 21 October 2013 Accepted: 30 January 2014 Published: 19 February 2014

\section{References}

1. Landelijke Stuurgroep Multidisciplinaire Richtlijnontwikkeling in de GGZ: Multidisciplinary guideline depression: guideline for diagnostics and treatment of adult clients with a major depressive disorder. Utrecht: Trimbos-instituut: 2005 [in Dutch].

2. Landelijke Stuurgroep Multidisciplinaire Richtlijnontwikkeling in de GGZ: Revision of the Multidisciplinary guideline for Depression. Guideline for the 
diagnosis, treatment and care of adult patients with a depressive disorder. Utrecht: Trimbos-instituut; 2010 [in Dutch]

3. National Collaborating Centre for Mental Health: Depression: the treatment and management of depression in adults: NICE clinical guideline 90. London: National Institute for Health and Clinical Excellence; 2009.

4. New Zealand Guidelines Group: Identification of common mental disorders and management of depression in primary care. Wellington: New Zealand Guidelines Group; 2008.

5. Whitty P, Gilbody S: NICE, but will they help people with depression? The new National Institute for Clinical Excellence depression guidelines. Br J Psychiatry 2005, 186:177-178.

6. Kessler RC, Berglund P, Demler O, Jin R, Koretz D, Merikangas KR, Rush AJ, Walters EE, Wang PS: The epidemiology of major depressive disorder: results from the National Comorbidity Survey Replication (NCS-R). JAMA 2003, 289(23):3095-3105

7. Smolders M, Laurant M, Verhaak P, Prins M, van Marwijk H, Penninx B, Wensing $M, G r o l$ R: Adherence to evidence-based guidelines for depression and anxiety disorders is associated with recording of the diagnosis. Gen Hosp Psychiatry 2009, 31(5):460-469.

8. Nuijen J, Van Dijk CE, Verhaak PFM, Verheij RA: Trendreport Mental Healthcare. In Trendreport Mental Healthcare 2010, part 2. Access and consumption of care. Edited by Nuijen J. Utrecht: Trimbos-instituut; 2010:181-235 [in Dutch].

9. Helgason T, Tomasson H, Zoega T: Antidepressants and public health in Iceland. Time series analysis of national data. Br J Psychiatry 2004, 184:157-162.

10. Hemels ME, Koren G, Einarson TR: Increased use of antidepressants in Canada: 1981-2000. Ann Pharmacother 2002, 36(9):1375-1379.

11. McManus P, Mant A, Mitchell PB, Montgomery WS, Marley J, Auland ME: Recent trends in the use of antidepressant drugs in Australia, 1990-1998. Med J Aust 2000, 173(9):458-461.

12. Morrison J, Anderson MJ, Sutton M, Munoz-Arroyo R, McDonald S, Maxwell M, Power A, Smith M, Wilson P: Factors influencing variation in prescribing of antidepressants by general practices in Scotland. Br J Gen Pract 2009, 59(559):e25-e31.

13. Olfson M, Marcus SC, Druss B, Elinson L, Tanielian T, Pincus HA: National trends in the outpatient treatment of depression. JAMA 2002, 287(2):203-209.

14. Stichting Farmaceutische Kengetallen: Antidepressant use increases strongly. Pharm Weekb/ 2005, 140(23):10 [in Dutch].

15. Moore M, Yuen HM, Dunn N, Mullee MA, Maskell J, Kendrick T: Explaining the rise in antidepressant prescribing: a descriptive study using the general practice research database. BMJ 2009, 339:b3999.

16. Piek E, van der Meer K, Hoogendijk WJ, Penninx BW, Nolen WA: Most antidepressant use in primary care is justified; results of the Netherlands Study of Depression and Anxiety. PLoS One 2011, 6(3):e14784.

17. Van Marwijk HW, Bijl D, Ader HJ, de Haan M: Antidepressant prescription for depression in general practice in The Netherlands. Pharm World Sci 2001, 23(2):46-49.

18. Spies T, Mokking H, de Vries RP, Grol R: GP often chooses antidepressants independent of depression severity. Huisarts Wet 2004, 47(8):364-367 [in Dutch].

19. Zwaanswijk M, Verhaak PFM: Effective brief interventions for psychological problems; a synthesis of the evidence on applicability in general practice. Utrecht: Nivel; 2009 [in Dutch].

20. Prins MA, Verhaak PFM, Bensing JM, van der Meer K: Health beliefs and perceived need for mental health care of anxiety and depression-the patients' perspective explored. Clin Psychol Rev 2008, 28(6):1038-1058.

21. Van Geffen EC, van der Wal SW, van Hulten R, de Groot MC, Egberts AC, Heerdink ER: Evaluation of patients' experiences with antidepressants reported by means of a medicine reporting system. Eur J Clin Pharmacol 2007, 63(12):1193-1199.

22. Van Rijswijk $E$, van Hout $H$, van de Lisdonk $E$, Zitman F, van Weel C: Barriers in recognising, diagnosing and managing depressive and anxiety disorders as experienced by Family Physicians; a focus group study. BMC Fam Pract 2009, 10:52.

23. Van Schaik DJ, Klijn AF, van Hout HP, Van Marwijk HW, Beekman AT, de Haan M, van Dyck R: Patients' preferences in the treatment of depressive disorder in primary care. Gen Hosp Psychiatry 2004, 26(3):184-189.

24. Van der Feltz-Cornelis CM: Towards integrated primary health care for depressive disorder in the Netherlands. The depression initiative. Int $J$ Integr Care 2009, 9:e83.

25. Chin $\mathrm{MH}$ : Quality improvement implementation and disparities: the case of the health disparities collaboratives. Med Care 2010, 48(8):668-675.
26. Katzelnick DJ, Von KM, Chung H, Provost LP, Wagner EH: Applying depression-specific change concepts in a collaborative breakthrough series. Jt Comm J Qual Patient Saf 2005, 31(7):386-397.

27. Øvretveit J, Bate P, Cleary P, Cretin S, Gustafson D, Mclnnes K, McLeod H, Molfenter T, Plsek P, Robert G, Shortell S, Wilson T: Quality collaboratives: lessons from research. Qual Saf Health Care 2002, 11(4):345-351.

28. Schouten LM, Hulscher ME, van Everdingen JJ, Huijsman R, Grol RP: Evidence for the impact of quality improvement collaboratives: systematic review. BMJ 2008, 336(7659):1491-1494.

29. Franx G, Meeuwissen JA, Sinnema H, Spijker J, Huyser J, Wensing M, de Lange J: Quality improvement in depression care in the Netherlands: the Depression Breakthrough Collaborative. A quality improvement report. Int J Integr Care 2009, 9:e84.

30. Verheij RA, van Dijk CE, Stirbu-Wagner I, Abrahamse H, Davids R, Braspenning J, Van Althuis T, Korevaar JC: National Information Network General Practice: facts and numbers about Dutch general practice. Utrecht/Nijmegen: NIVEL/IQ; 2009 [in Dutch].

31. Lamberts HWM: International Classification of Primary Care (ICPC). Oxford: Oxford University Press; 1990

32. Nuijen J, Volkers AC, Verhaak PFM, Schellevis FG, Groenewegen PP, van den Bos GA: Accuracy of diagnosing depression in primary care: the impact of chronic somatic and psychiatric co-morbidity. Psychol Med 2005, 35:1185-1195

33. WONCA: International Classification of Health Problems in Primary Care (ICHPPC-2-Defined). Oxford: Oxford University Press; 1983.

34. Berwick DM: Developing and testing changes in delivery of care. Ann Intern Med 1998, 128(8):651-656.

35. Hulscher M, Schouten L, Grol R: Quest for Quality and Improved Performance: Collaboratives. London: The Health Foundation; 2009.

36. Franx G, Spijker J, Huyser J, de Doelder P: Reduction in depression: Breakthrough method reduces over treatment. Medisch Contact 2006 61(40):1592-1595.

37. Meeuwissen JA, van der Feltz-Cornelis CM, van Marwijk HW, Rijnders PB, Donker MC: A stepped care programme for depression management: an uncontrolled pre-post study in primary and secondary care in The Netherlands. Int J Integr Care 2008, 8:e05.

38. Franx $G$, Oud M, de Lange J, Wensing M, Grol R: Implementing a stepped-care approach in primary care: results of a qualitative study. Implement Sci 2012, 7:8.

39. Danz MS, Rubenstein LV, Hempel S, Foy R, Suttorp M, Farmer MM, Shekelle PG: Identifying quality improvement intervention evaluations: is consensus achievable? Qual Saf Health Care 2010, 19(4):279-283.

40. Wagner EH, Glasgow RE, Davis C, Bonomi AE, Provost L, McCulloch D, Carver P, Sixta C: Quality improvement in chronic illness care: a collaborative approach. Jt Comm J Qual Improv 2001, 27(2):63-80.

41. Meredith LS, Mendel P, Pearson M, Wu SY, Joyce G, Straus JB, Ryan G, Keeler E, Unützer J: Implementation and maintenance of quality improvement for treating depression in primary care. Psychiatr Serv 2006, 57(1):48-55.

42. Smith MJ: "Doing Well": an initiative to improve depression care [MD Thesis]. Glasgow: University of Glasgow; 2010.

43. Richards DA, Bower P, Pagel C, Weaver A, Utley M, Cape J, Pilling S, Lovell K, Gilbody S, Leibowitz J, Owens L, Paxton R, Hennessy S, Simpson A, Gallivan S, Tomson D, Vasilakis C: Delivering stepped care: an analysis of implementation in routine practice. Implement Sci 2012, 7:3.

44. Clark DM: Implementing NICE guidelines for the psychological treatment of depression and anxiety disorders: The IAPT experience. Int Rev Psychiatry 2011, 23:318-327.

45. Frank RG, Huskamp HA, Pincus HA: Aligning incentives in the treatment of depression in general practice with evidence-based practice. Psychiatr Serv 2003, 54(5):682-687.

46. Verhaak PFM, Ten Have JJM, Scholte MM: Competition in psychological care. Medisch Contact 2007, 40(2):1642-1645 [in Dutch].

47. Craig P, Dieppe P, Macintyre S, Michie S, Nazareth I, Petticrew M: Developing and evaluating complex interventions: the new Medical Research Council guidance. BMJ 2008, 337:a1655.

doi:10.1186/1471-2296-15-35

Cite this article as: Franx et al:: Implementing guidelines for depression on antidepressant prescribing in general practice: a quasi-experimental evaluation. BMC Family Practice 2014 15:35. 